\title{
CORPUS Corpus
}

15 | 2016

Corpus de français parlé et français parlé des corpus

\section{Le CFPP2000 : constitution, outils et analyses. Le} cas des interrogatives indirectes

The spoken Parisian French corpus in the 2000': constitution, tools and analyses. The case of indirect interrogatives clauses

Sonia Branca-Rosoff et Florence Lefeuvre

\section{OpenEdition}

Journals

Édition électronique

URL : http://journals.openedition.org/corpus/3043

ISSN : 1765-3126

Éditeur

Bases ; corpus et langage - UMR 6039

Édition imprimée

Date de publication : 15 octobre 2016

ISSN : 1638-9808

Référence électronique

Sonia Branca-Rosoff et Florence Lefeuvre, « Le CFPP2000 : constitution, outils et analyses. Le cas des interrogatives indirectes », Corpus [En ligne], 15 | 2016, mis en ligne le 15 janvier 2017, consulté le 08 septembre 2020. URL : http://journals.openedition.org/corpus/3043

Ce document a été généré automatiquement le 8 septembre 2020

(C) Tous droits réservés 


\section{Le CFPP2000 : constitution, outils et analyses. Le cas des interrogatives indirectes}

The spoken Parisian French corpus in the 2000': constitution, tools and analyses. The case of indirect interrogatives clauses

Sonia Branca-Rosoff et Florence Lefeuvre

1 L'objet de cet article est de préciser quel type de français apparaît dans le Corpus de français parlé parisien des années 2000, constitué par Sonia Branca-Rosoff, Serge Fleury, Florence Lefeuvre et Mat Pirès ${ }^{1}$. Ce corpus atteint, en mars 2015, 602000 mots pour 42,5 heures d'enregistrement, réparties en 32 interviews, qui couvrent la plupart des arrondissements de l'est et du centre de Paris ainsi que quelques villes de la petite couronne. La diversité des locuteurs interrogés doit permettre de préciser l'ampleur de la variation observable dans cet usage oral du français contemporain, que nous appelons la langue commune. Dans cet article, nous nous appuierons sur l'exemple des interrogatives indirectes (percontatives) pour situer ce registre qui ne se confond ni avec le français standard, tel qu'il est décrit dans les grammaires et les dictionnaires, ni avec les variétés les plus vernaculaires.

\section{L'hypothèse de la langue commune}

Les choix qui ont présidé à la confection du CFPP2000 expliquent les formes de français parlé observables dans le corpus. Le CFPP2000 est un corpus d'entretiens semi-préparés (l'enquêteur disposant d'une grille de questions qui n'a pas été communiquée auparavant à l'enquêté) sur le thème du rapport des locuteurs à leur quartier et à la ville de Paris en général. Ce corpus ne relève donc pas de l'observation participante, où l'enquêteur cherche à disparaître pour ne pas modifier les échanges ordinaires du groupe qu'il observe. Les universitaires qui ont réalisé l'enquête n'ont pas davantage adopté «la neutralité » distante, recommandée un temps, notamment en sociologie, 
puisqu'ils n'hésitent pas à s'impliquer dans l'échange, ce qui rapproche les entretiens du CFPP2000 de «vrais » dialogues. L'enquêteur adopte une attitude empathique, mais il pousse parfois ses interlocuteurs dans leurs retranchements, rappelant ainsi qu'il est extérieur à leur groupe. Ce dispositif, marqué par l'asymétrie des pôles d'interlocution, a certainement des conséquences en ce qui concerne la production des opinions ; ainsi, lorsqu'il s'agit d'immigration, les enquêtés tiennent compte des positions défavorables à la critique des migrants qu'ils prêtent à l'enquêteur.

3 Ce dispositif a aussi des conséquences sur les activités langagières développées lors de l'entretien. Il entraîne, par exemple, le recours à des séquences argumentatives, car le locuteur, qui ne peut compter sur une étroite connivence avec l'enquêteur, entre souvent dans l'exposé de ses motifs et plus généralement dans un discours de justification. De même, il comporte une sur-utilisation des marques de réflexivité 2 . La volonté d'agir sur le point de vue de l'enquêteur s'accompagne, chez beaucoup d'enquêtés, de retours sur leurs formulations comme chez cette Ivryenne des « classes moyennes", soucieuse de n'être pas confondue avec la "racaille» des cités et de préserver les avantages procurés par une école d'élite, mais qui vit dans une banlieue communiste dont elle partage en principe les options généreuses :

(1) on faisait partie de l'école + entre guillemets privilégiée d'Ivry hein ( $\mathrm{mm})$ de toute façon ça faut faut que ça soit bien clair $(\mathrm{mm})+$ et euh nos enfants avaient une un très bon niveau qui se vendait très bien sur Paris + nous on peut dire notre fils on l'a vendu sur son livret scolaire hein c'est + on l'a vendu dans trois collèges parisiens [IV-02]

4 La locutrice revient sur la brutalité de l'étiquette «école privilégiée » qu'elle pourrait attribuer à une fiction d'énonciateur tenant du politiquement correct - soit l'enquêtrice, soit le public virtuel des lecteurs de l'interview - avant de proposer un « très bon niveau » qui induit une orientation argumentative moins négative.

Le dispositif d'enquête a aussi des effets en ce qui concerne le registre, question centrale, lorsqu'il s'agit des interrogatives indirectes. Les locuteurs n'usent pas librement de la variété qu'ils emploient dans leur milieu vernaculaire, si celle-ci s'écarte sensiblement de la variété utilisée par l'enquêteur. Les traits pouvant être perçus comme trop marqués sont inhibés au profit de traits où langue de l'interviewé et langue de l'intervieweur convergent ${ }^{3}$ : enquêtés et enquêteur vont avoir recours à une «langue commune ». Pour désigner cette variété, nous aurions pu utiliser la notion de standard très employée en sociolinguistique, mais les entretiens s'en distinguent sur de nombreux points. Nous conservons le terme standard pour renvoyer aux normes sociales instituées, telles qu'on les trouve dans les grammaires et les dictionnaires. De ce fait, le terme ne recouvre pas les normes émergentes déviantes que l'on rencontre dans le corpus CFPP2000 - comme nous le montrerons à propos des interrogatives indirectes. Le corpus ne peut davantage servir à décrire les différents vernaculaires (français dit " des cités », jeux bilingues...) ou les argots de métiers qui circulent dans la capitale. Il est centré sur la variété qui émerge dans la situation d'intercommunication cadrée comme «Entretiens-sur-la-ville-de-Paris-recueillis-par-des-universitaires-etdestinés-à-être-mis-en-ligne ", une variété que les enquêtés jugent convenable dans une situation où ils sont bien avertis que leurs discours seront diffusés dans l'espace public, puisqu'ils doivent signer une autorisation de mise en ligne après anonymisation. Pour résumer, le corpus permet d'aborder le comportement des locuteurs, en se référant non pas aux prescriptions des grammaires et des dictionnaires (il s'agit 
justement d'en mesurer l'efficacité) ou aux déclarations subjectives de ces locuteurs (on sait qu'elles sont souvent très normatives), mais en dégageant des "normes descriptives ", statistiques, qui correspondent à une description objective des comportements des locuteurs. Dans la variété de CFPP2000, il s'agit d'un français oral, délesté d'un certain nombre de traits communautaires, l'accommodement réalisé entre les deux partenaires aboutissant à une variété qui présente une homogénéité suffisante pour qu'il y ait un échange. Plutôt que d'une variété, il faudrait d'ailleurs parler d'un espace de variation, où les locuteurs se rapprochent tantôt du pôle familier, tantôt du pôle standard. Le corpus implique actuellement 58 locuteurs ${ }^{4}$. C'est dire qu'il n'est pas encore parvenu au stade de la représentativité statistique. Toutefois, il ouvre sur la diversité des usages.

7 Les métadonnées permettent de tenir compte de la variation due à la situation sociale des locuteurs. Ces renseignements a priori peuvent être utilement complétés par la lecture de l'entretien afin de dégager les identités sociolinguistiques revendiquées par les locuteurs qui peuvent influencer leurs comportements.

8 Le corpus CFPP2000 permet par conséquent d'observer la diffusion d'une forme en tenant compte du sexe, de l'âge et du statut des locuteurs. Dans un corpus synchronique, une progression en cours se repère notamment aux emplois plus nombreux dans la jeune génération que dans la génération ancienne. Labov (1976) parle à ce propos de changement en temps apparent.

\section{Les percontatives ou interrogatives indirectes: critères de reconnaissance}

Dans ce travail, nous laissons de côté le problème des rapports entre questions, propositions relatives et interrogations indirectes. Nous ne discutons pas des choix théoriques impliqués par l'usage de telle ou telle terminologie. Nous adopterons celle de Le Goffic (1993), qui parle de percontatives. Nous savons bien que chaque terme renvoie à une définition différente de ce type de structures, mais dans la mesure où le lecteur peut identifier ce dont nous parlons, ce choix n'a pas paru trop gênant. Trois critères de reconnaissance ( $c f$. Lefeuvre 2006) nous ont permis de relever les percontatives dans le CFPP2000 : le type de verbes introducteur, le type de mots en quet le rôle de la préposition.

\subsection{Une approche sémantique : le verbe introducteur}

D'après R. Martin 1984, le verbe introducteur doit évoquer un univers de croyance et la non-explication de la valeur de $p$ dans la subordonnée ${ }^{5}$. Pour G. Serbat (1985:9) :

Le verbe introducteur n'a pas besoin d'être lui-même percontatif. Il suffit qu'il soit compatible avec la conception ou l'énoncé d'une donnée problématique autrement dit il suffit qu'il appartienne aux classes larges « savoir » ou « dire ».

11 Le Goffic (p. 265), quant à lui, retient trois types de verbes : i) verbe d'action sur autrui (demander), ii) verbes déclaratifs et iii) verbes de connaissance :

(2) les gens m'ont beaucoup demandé quand il est mort [14-01]

(3) donc je lui ai dit où il était [IV-01]

(4) je sais pas où c'est [03-01]

12 B. Defrancq (2005, p. 170), signale, en repartant de la liste des verbes introducteurs donnée dans L. Karttunen 1978, que les interrogatives indirectes «s'associent toutes à 
des verbes référant d'une façon ou d'une autre à un procès qui a trait à l'information ", même si «l'inverse n'est pas vrai : tous les verbes de ce type ne s'associent pas à une [interrogative enchâssée] ».

\subsection{Les mots en qu-}

13 Il se révèle parfois difficile de distinguer les percontatives des intégratives qui regroupent relatives sans antécédent et circonstancielles en comme, quand, si, que. Pour s'assurer qu'il s'agit bien d'une percontative, les grammairiens ont proposé différents tests de substitution dont nous rappelons quelques-uns ci-dessous.

\subsection{1 comment $\neq$ comme}

D'après P. Le Goffic, le verbe introducteur percontatif accepte des subordonnées en comment et non en comme (1993: 45). Ainsi pour :

(5) je sais pas où c'est [03-01]

où peut être remplacé par comment et non par comme :

(6) Je sais pas comment c'est / ${ }^{*}$ comme c'est

Ce qui montre que savoir se construit bien avec des percontatives.

\subsection{2 quel}

17 Le verbe introducteur percontatif accepte des subordonnées en quel (Le Goffic $1987: 84)^{6}$ :

(7) je sais pas quel est cet individu

\subsection{3 insertion de est-ce que}

M. Pierrard ajoute le test de l'insertion de est-ce que dans la subordonnée. Si l'insertion est possible, il s'agit d'une percontative (« interrogative indirecte », $1988: 32$ ) :

(8) je sais pas où on irait jouer au foot [KB-01]

(9) je sais pas où est-ce qu'on irait jouer au foot

\subsection{L'intégrative et la préposition impliquée}

19 L'intégrative (relative sans antécédent) dépend de la préposition impliquée par le verbe introducteur, à la différence de la percontative ou interrogative indirecte :

(10) Je pense à qui tu sais (relative sans antécédent, ex. tiré de Léard 1992)

(11) je ne sais $(\mathrm{mm})$ même pas de quoi ils vivaient (interrogative indirecte, CFPP2000 [14-02])

Cela dit, il existe des structures intégratives dont la préposition dépend bien du verbe de la subordonnée :

(12) C'est à quoi je pense (ex. tiré de Lefeuvre 2006 : 151)

21 D'autre part, dans le discours informel, ces tests sont moins pertinents. Ainsi peut-on trouver est-ce que dans des subordonnées qui ne sont pas ici des percontatives mais des intégratives (ou relatives sans antécédent) basées sur la structure de clivées :

(13) je suis déjà allé où est-ce que j'ai envie d'aller [SO-02]

(14) tu entendras pas quelqu'un dire : "c'est qu'est-ce que je t'ai dit" [12-02] 
(15) je pensais plutôt à qu'est-ce qui se passe dans la cour de récréation [11-02]

En effet, les verbes aller, être, penser n'introduisent pas de percontatives et ne peuvent pas se construire avec quel :

(16) *Je suis déjà allé dans quel lieu tu es allé

*C'est dans quel lieu tu es allé

* Je pensais à quel lieu tu es allé

En outre, certains verbes comme parler peuvent être pris comme des verbes introducteurs de percontatives, alors qu'ils ne le sont pas dans un discours standard. C'est ce que l'on a dans cet exemple qui renferme est-ce que et qui est proféré d'un seul tenant, sans pause ni décrochage intonatif, ce qui aurait pu faire penser à un abandon de construction et un redémarrage avec une interrogative directe. Il faut donc considérer qu'il s'agit d'une subordonnée dépendant du verbe parler et introduite par comment :

(17) parler comment est-ce qu'on vit euh + comment est-ce qu'on vit en tant que Parisienne avec euh voilà avec deux enfants [11-01]

D'après ces deux indices (présence de comment et de est-ce que), il s'agit d'une interrogative indirecte mais le verbe parler n'est pas un verbe reconnu comme un verbe introducteur de percontatives en français standard. Nous avons le choix entre deux types d'analyse : soit il s'agit d'un verbe qui est transformé en verbe introducteur de percontative, proche de dire, soit il s'agit d'une subordonnée qui est une intégrative adverbiale (circonstancielle en comment), paraphrasable par de la façon dont on vit (cf. Lefeuvre 2009).

Voyons à présent quels types de percontatives figurent dans le CFPP2000.

\section{Les types de percontatives dans le CFPP2000}

Cinq types d'interrogatives indirectes apparaissent dans le CFPP, avec des fréquences variées.

\subsection{Les percontatives attendues selon les grammaires du français standard}

Il s'agit des percontatives qui se présentent sans inversion, sans est-ce que, avec le mot en qu- en début de subordonnée, comme elles sont décrites par exemple dans Riegel et al. 2009 (838).

Deux schémas sont attestés dans le CFPP. Le schéma S-V :

(18) je sais pas où c'est chez (Özgur [H 32 ans, élevé dans le $3^{\mathrm{e}}$ arrondissement, 03-01])

(19) et et quand on sait où d'où on vient on se dit "je suis là j'ai beaucoup de chance"

[Pierre-Marie Simo, H 34 ans, Camerounais d'origine, arrivé en France, dans le $18^{\mathrm{e}}$

arrondissement populaire, à 9 ans, 18-01]

Et le schéma avec postposition du GN sujet :

(20) je sais pas où est la préfecture [SBR enq, F, 65 ans]

Nous n'avons pas relevé toutes les structures standard car leur décompte aurait nécessité de faire des requêtes longues pour chaque verbe introducteur, mais elles sont bien attestées et chez des locuteurs d'origine sociale variée (cf. plus bas le sondage pour les percontatives introduites par je (ne) sais pas). 
31 Il y a ainsi 11 occurrences de je ne sais pas ce que chez des locuteurs jeunes comme Paul Simo, âgé de 18 ans (je sais pas ce que je vais faire, [18-01]), et chez des locuteurs âgés comme J. Pelletier qui vit en banlieue et a un faible bagage scolaire (je sais pas ce que la vie me réserve [IV-01]).

\subsection{Les percontatives en est-ce que} dans les usages des locuteurs parisiens. Le CFPP2000 comporte 27 percontatives en estce que. Les verbes introducteurs se répartissent dans les trois catégories signalées plus haut :

- type demander (4 exemples) :

(21) ils me demandent un peu euh + + où est-ce que j'aime bien aller dîner machin et tout + (Raphaël Larivière, H. 23 ans, vit dans le $7^{\mathrm{e}}$, quartier le plus bourgeois de Paris, étudiant en médecine [07-04])

- type dire, énonciation (3 exemples) :

(22) vous ne signalez pas qu'est-ce que vous faites (Thérèse Le Vern, F 70 ans, enfance jusqu'à 10 ans, île de la Réunion [12-03])

- type savoir, épistémique (20 exemples) :

(23) euh c'est vrai que quand on s'cachait derrière les voitures on prenait rapidement l'réflexe de regarder où est-ce qu'on se cachait (Özgur, H. 32 ans, ingénieur du son pour l'audiovisuel, élevé à Paris dans le $3^{\mathrm{e}}$ arr. [03-01])

Le verbe introducteur le plus fréquent est savoir (11 occurrences), qui apparaît massivement avec la négation: 7 je sais pas, 2 je sais plus; contre seulement 2 savoir affirmatifs, comme dans l'exemple cité plus haut. L'affinité entre la négation et la structure en est-ce que est un bon indicateur d'une probable influence de l'interrogative indépendante. La forme affirmative $\mathrm{du}$ verbe savoir n'a pas de signification interrogative et, dans ce cas, le locuteur a tendance à ne pas s'aligner sur l'interrogative directe en est-ce que. Au contraire, lorsque, sémantiquement, ne pas savoir exprime une question, le locuteur adopte davantage la structure syntaxique en est-ce que, caractéristique de la question.

Même si cette structure n'est pas répertoriée dans les grammaires ou est considérée comme ne relevant pas du bon usage (Riegel et al., 2009: 838), elle existe depuis au moins quatre siècles. De grands auteurs du XVII ${ }^{\mathrm{e}}$ siècle en ont usé sans soulever la réprobation des puristes de l'époque :

(24) Il faut voir de quoi est-ce qu'elle est malade (Molière, Le Médecin malgré lui)

(25) me demandant qu'est-ce que c'était que des tambours qui battaient (Cardinal de Retz, ex. tiré de Lefeuvre 2014)

(26) Dites-nous qui est-ce que nous pouvons choisir pour notre roi (Fénelon, ex. tiré de Lefeuvre 2014)

Plusieurs linguistes, comme B. Defrancq (2000, p. 135 pour Corpaix) ou C. Blanche Benveniste (1997, p. 41), signalent que la structure est bien attestée dans d'autres corpus. C. Blanche Benveniste estime même qu'on ne devrait plus parler de faute à son propos. Dans le même sens, nous notons que, sauf exception ${ }^{7}$, les locuteurs, en usent sans se corriger. Le type "standard» est cependant le plus fréquent, ce que permet d'observer la comparaison d'énoncés introduits par je (ne) sais pas, structure dont nous avons dit qu'elle favorise est-ce que : 
Tableau 1. Fréquence des percontatives

\begin{tabular}{|l|l|l|}
\hline & $\begin{array}{l}\text { Percontatives suivant l'ordre S-V } \\
\text { (ou VS) }\end{array}$ & $\begin{array}{l}\text { Percontatives renfermant est- } \\
\text { ce qu- }\end{array}$ \\
\hline je (ne) sais pas qui & 4 & 1 \\
\hline je (ne) sais pas où & 7 & 3 \\
\hline je (ne) sais pas pourquoi & 8 & 0 \\
\hline je (ne) sais pas comment & 23 & 0 \\
\hline je (ne) sais pas ce qu- /qu'est- \\
ce qu-
\end{tabular}
un exemple avec l'introducteur je sais même plus :

(27) je sais même plus quand est-ce qu'il est devenu à la mode [Anita Musso, 46 ans, licence, auxiliaire de vie a grandi dans le $\left.11^{\mathrm{e}}, 11-01\right]$

Il faut noter l'influence des mots en qu-. Où favorise l'emploi de est-ce que. Comment et pourquoi l'inhibent.

\subsection{Les percontatives qui renferment deux mots en qu-}

41 Seulement deux exemples de ce type ont été répertoriés, tous avec comment :

(28) je sais pas comment que ça se passe dans les autres pays + mais je trouve qu'en (Valentine Testanier, 60 ans, [12-03])

(29) moi ce qui m'intéressait et ce qui m'intéresse toujours + + c'est + l'humain + le vécu des gens $(\mathrm{mm})$ savoir comment qu'on va pouvoir les + les sortir de cette foutue merde + où on est en ce moment $(\mathrm{mm})$ (Jacqueline Pelletier, 65 ans, [IV-01])

Nous n'avons pas trouvé d'exemples en quand qu- ni où qu-. Notons que ce schéma est également rare en ce qui concerne les interrogatives (directes). Nous n'en avons trouvé dans le CFPP que cinq, produites par le même locuteur ( $c f$. Lefeuvre \& Rossi-Gensane, 2015) :

(30) comment que c'était j'en sais rien (Marie-Hélène Matera, 67 ans, secrétaire retraitée, enfant d'immigrés italien, [MO-02])

(31) et ben y a eu euh euh comment qu'il s'appelle + aidez-moi Mireille (Marie-Hélène Matera, [MO-02])

(32) + qui fabriquait bon euh comment que ça s'appelle ben des bougies (Marie-Hélène Matera, [MO-02])

(33) dans les dans les autres unités par exemple dans la distribution ou dans: comment que ça s'appelle la distribution puis l'autre c'est quoi déjà ? bougies (Marie-Hélène Matera, [MO-02])

(34) si un souvenir me me me tente tu sais des fois un vieux moulin à la main là comment que ça s'appelle (Marie-Hélène Matera, [MO-02]) 
Dagnac (2014) rappelle la forte stigmatisation de ce type de structure qui serait en déclin. Defrancq (2005) n'évoque pas ce schéma pour les percontatives. Ce schéma semble bien inscrit en langue, puisque l'on peut trouver quelques percontatives en comment que à l'oral représenté ( 3 occurrences de sais pas comment que, toutes 3 sur la première moitié $\mathrm{du} \mathrm{xx}^{\mathrm{e}}$ siècle) :

(35) J'sais pas comment que tu te démerdes, mais t'es toujours d'attaque! (Frantext, Genet, le Miracle de la rose, 1947)

(36) Je ne sais pas comment que ça se fait. (Frantext, Péguy, Le Mystère de la charité de Jeanne d'Arc, 1910)

Ce type de percontative est attesté sur Frantext à partir de 1879,

(37) Dis-moi un peu comment que tu t'y prendrais! (Huysmans, Les Sours Vatard, 1879) les dernières occurrences trouvées datant de 1966 :

(38) Ceux d'entre nous qui avaient encore la force de parler se soulevèrent sur un coude et demandèrent comment que ça s'était passé. (Pérec, Quel Petit Vélo à guidon chromé au fond de la cour?, 1966)

On peut donc faire l'hypothèse que ce schéma, pour les percontatives, est effectivement en déclin.

En revanche, il est davantage présent dans le CFPQ (corpus de français parlé québécois de 471575 mots) ; pour les interrogatives (directes), 18 exemples sur les 50 relevés avec comment en position frontale adoptent cette structure ( $c f$. Lefeuvre \& Rossi-Gensane, 2015) :

(39) comment qu'ils appellent ça dans les bu un bureau de travail/ là dans les:::: (CFPQ, sous- corpus $15, \mathrm{CFPQ}$ )

et nous avons trouvé 11 exemples de percontatives amorcées par comment que :

(40) hum hum hum hum hum hum hum et pour dire hein que tu sais pas comment que ça ça peut brimer un enfant (sous-corpus 20, CFPQ)

\subsection{Les percontatives avec inversion}

\section{F. Gadet $(1997,108)$ relève des énoncés comme :}

(41) Je me demande quand part-il

Ce type d'énoncés avec inversion semble typique de conduites d'hypercorrection. Le locuteur en « fait trop » : il essaie de produire une forme d'interrogation qui relève d'un usage soutenu en inversant le verbe et le sujet, mais en alignant la percontative sur la forme la plus normée d'interrogative, il oublie qu'il n'y a pas d'inversion dans la subordonnée. De fait, on entend parfois ces formes à la radio ou à la télévision et on les trouve à l'écrit dans des copies d'étudiant. Dans la situation d'enregistrement du CFPP2000, les locuteurs n'ont pas jugé utile d'avoir recours au registre le plus soutenu. De fait ces structures ne sont pas attestées.

\subsection{Les percontatives avec le mot en qu- in situ}

Nous avons relevé seulement deux exemples de percontatives in situ dans le CFPP2000, et tous les deux chez des locuteurs d'origine maghrébine qui vivent dans des cités ghettoïsées de la même banlieue populaire, Saint-Ouen :

(42) euh il me dit "ben écoute t'as encore trois trois autres entretiens entretien médical psychologique et un autre entretien je sais plus c'était quoi la fin et plus un test un test [ $\mathrm{mm}$ ] euh + un test un questionnaire (Youcef, 29 ans, actuellement sans emploi, titulaire d'un master de géographie, [SO-02]) 
(43) non juste le le le le montrer euh euh la la la vie c'est quoi euh de se lever à six heures [mm] du matin récurer les toilettes avec les brosses à dents euh (Abdel, 25 ans, manutentionnaire au journal Le Parisien, CFPP2000, [SO-02])

\section{Analyse des données} deux variantes de percontatives qu'ils traitent comme appartenant toutes les deux à la langue commune. Les interrogatives indirectes en est-ce que ne sont pas cantonnées à un type de locuteur. On les trouve par exemple chez R. Larivière, un étudiant en médecine de 23 ans, qui habite le quartier le plus bourgeois de Paris, le $7^{\mathrm{e}}$ :

(44) Je sais pas où est-ce que je vais

et dans la bouche de M. L. Orsin, qui a grandi dans le $11^{\mathrm{e}}$ (un quartier assez populaire), mais qui est fille d'instituteur et qui est elle-même devenue professeure des écoles, métier en rapport avec la transmission des normes linguistiques :

(45) Je sais pas où est-ce que vous auriez été.

\subsection{Les formes in situ et l'influence des français périphériques}

En revanche, ce n'est pas le cas des percontatives in situ. Les deux formes répertoriées proviennent de locuteurs jeunes qui ont grandi dans la même banlieue défavorisée :

(46) euh il me dit "ben écoute t'as encore trois trois autres entretiens entretien médical psychologique et un autre entretien je sais plus c'était quoi la fin et plus un test un test [mm] euh + un test un questionnaire (Youcef, 29 ans, actuellement sans emploi, titulaire d'un master de géographie, [SO-02])

(47) non juste le le le le montrer euh euh la la la vie c'est quoi euh de se lever à six heures [mm] du matin récurer les toilettes avec les brosses à dents euh (Abdel, 25 ans, manutentionnaire au Parisien [SO-02])

Des exemples relevés à la volée montrent qu'il s'agit d'une structure que l'on entend chez d'autres locuteurs d'origine maghrébine. L'exemple suivant est prononcé par une femme de ménage d'origine algérienne dans un entretien en cours de transcription :

(48) quand il manque des bonbons vous savez c'est qui (adulte, Samia, origine

algérienne, 49 ans, scolarité jusqu'en $6^{\mathrm{e}}$, arrivée en France à 22 ans)

En français hexagonal, cette structure apparaît chez de jeunes enfants, mais a toutes les chances de disparaître par la suite, lorsqu'ils appartiennent à des milieux favorisés :

(49) tu sais son nom de famille c'est quoi (enfant d'enseignant, 4 ans)

(50) je sais c'est qui mes copines (enfant d'enseignant, 8 ans)

Elle a cependant été observée chez des élèves plus âgés qui sont scolarisés dans des établissements classés en Zone d'éducation prioritaire (Paris, CM2 / $6^{\mathrm{e}}$ ). On en trouve de nombreuses attestations dans un corpus rassemblé par B. Lesort (2002) qui a été recueilli dans une école très ghettoïsée de Paris : presque tous les parents des classes observées viennent d'Afrique Noire, d'Asie ou du Maghreb. La plupart des percontatives utilisées par les enfants (prononcées sans pause, ni rupture intonative d'aucune sorte) sont des formes in situ :

(51) je sais pas c'est quoi (Abdoulay)

(52) je sais pas c'est comment (Idriss)

(53) je sais pas c'est combien d'heures techno (Idriss)

(54) y'a piscine, techno, après je sais pas y a quoi là-bas (Idriss)

(55) je sais pas ça veut dire quoi (Malamine) 
(56) je demande à la maîtresse si j'ai pas compris, elle m'aide après elle me dit c'est quoi, après elle m'explique (Malamine) téléréalité qui, semble-t-il, ignore qui est Jean Valjean ${ }^{8}$, résume son registre de langue par une percontative in situ :

(61) www.public.fr ’. Les Anges de la téléréalité 4 : Nabilla: "Je connais Jean Valjean mais je sais pas c'est qui, c'est un clochard ?" stylisation syntaxique.

Un autre message montre bien la différence d'évaluation des deux formes, puisqu'un commentateur, qui se voit comme un redresseur du mauvais français, fustige l'emploi de « je sais pas c'est quoi » et, ce qui ne manque pas de piquant, semble recommander l'usage de " qu'est-ce », sans prendre soin de différencier indépendante et enchâssée :

(62) à propos de je sais pas c'est quoi: heavendoor ns Forumeur alchimiste, 49 a C'est la différence entre la langue écrite et parlé... C'est vrai que toutes les langues évoluent... pas toujours dans le bon sens d'ailleurs... Normalement à la place de "c'est quoi" nous devrions dire et écrire "Qu'est -ce ?" forme interrogative... (Coups de blues - FORUM Ados-Sante forum.ados.fr)

\subsection{Hypothèses}

\footnotetext{
Comment expliquer l'apparition des percontatives in situ, et quelle évolution future pouvons-nous prévoir? Nous pouvons proposer des hypothèses complémentaires. Et non exclusives.
} 


\subsubsection{Une évolution naturelle du français} interrogatives indépendantes où le syntagme interrogatif est in situ : 500 exemples de c'est quoi, de 1970 à nos jours vs 37 exemples de 1927 à 1970 et 1 seul exemple (1881) de 1600 à 1926 :

(63) C'est quoi, ton boulot? (Arnaud Claude, Qu'as-tu fait de tes frères ?, 2010, Frantext) étape qui suit logiquement le développement des interrogatives indépendantes in situ que le corpus Frantext permet de documenter surtout à partir de 1927 : on pourrait prévoir que, dans une échéance plus ou moins longue, s'installeront dans tous les registres du français des percontatives in situ.

Dans la lignée des explications de H. Frei (1920), on peut noter que l'alignement des percontatives sur les interrogatives directes va dans le sens d'une plus grande expressivité et d'une plus grande économie. La forme en est-ce que a l'intérêt d'être pragmatiquement marquée (et dans un premier temps) de produire un effet d'emphase ${ }^{9}$ ; d'autre part, les structures des percontatives s'alignent sur les structures des interrogatives (directes), ce qui permet aux locuteurs d'unifier leurs façons de poser des questions.

De même que les interrogatives en qu'est-ce que ont pu donner des percontatives en qu'est-ce que (cf. plus haut), de même les interrogatives in situ aboutiront logiquement à des percontatives in situ.

70 Les percontatives ne sont pas dans ce cas introduites par un mot subordonnant; l'absence d'élément introducteur "subordonnant" n'empêche pas la relation de dépendance entre la proposition enchâssée et le verbe introducteur mais leur subordination s'établit en discours. Cela peut suggérer que les percontatives canoniques, du type :

(64) bon on sait quand quelqu'un a besoin de quelque chose [IV-01]

71 s'établissent également en discours et que quand n'est pas en fait subordonnant dans cet emploi (cf. Le Goffic $1993: 43$ : «la subordonnée, enchâssée directement, conserv[e] un caractère proche d'une indépendante »).

\subsubsection{Renforcement du phénomène}

Ce développement récent est peut-être renforcé par l'immigration massive qui a eu lieu en France à partir des années 60. La présence de cette structure dans les français périphériques (français de la Réunion, du Québec, Cameroun) peut s'expliquer ellemême par une tendance à la parataxe plus forte dans les usages périphériques ou par l'influence qu'ils auraient reçue des français régionaux (mais les attestations manquent). 


\subsubsection{La question de la rapidité des changements en cours}

Nous serons prudentes pour faire des pronostics sur l'évolution. D'une part, la percontative en est-ce que et le tour normé coexistent depuis trois siècles au moins. Cette coexistence constitue un exemple de plus des variations sur la longue durée constatables dans toutes les langues. Le langage est par nature hétérogène et comporte beaucoup de variations semblables. Il n'y a donc pas de raison de supposer un effondrement proche et d'ailleurs, dans le CFPP2000, les occurrences normées semblent dominer.

Pour autant, l'usage des structures in situ - qui permettent elles aussi d'aligner les percontatives sur les structures directes - devrait se poursuivre. Le seul frein que l'on peut imaginer est celui de la pression scolaire. Jusqu'à récemment, l'institution scolaire cherchait à imposer des formes de subordination "classique » où un subordonnant marquait la frontière entre un élément introducteur et un élément enchâssé et indiquait la relation de dépendance entre les deux. Cet idéal est évidemment troublé par les cas où l'on observe une relation de dépendance entre une proposition enchâssée et le verbe dont elle dépend en l'absence d'élément introducteur, ce qui conduit jusqu'à aujourd'hui à stigmatiser ces formes.

\section{Conclusion}

En 2014, les interrogatives indirectes en « est-ce que » font partie du français commun que décrit CFPP2000. Les interrogatives indirectes in situ sont encore aux marges du système, comme l'indique le fait que leur usage suffit pour caricaturer un locuteur sur Internet.

\section{BIBLIOGRAPHIE}

Bell A. (1984). « Language Style as Audience Design », Language in Society 13 : 145-204.

Biloa E. Des traits syntaxiques et morphosyntaxiques du français au Cameroun, www.unice.fr/bcl/ ofcaf/27/BILOA.pdf.

Blanche Benveniste C. (1997). Approches de la langue parlée en français. Paris : Ophrys.

Branca-Rosoff S. (2015). « Dieu est dans les détails. L'expression entre guillemets et ses usages », in J. Angermuller \& G. Philippe (dir.) Analyse du discours et dispositifs d'énonciation. Autour des travaux de Dominique Maingueneau. Limoges.

Branca-Rosoff S., Fleury S., Lefeuvre F. \& Pires M. (2012). Discours sur la ville. Présentation du Corpus de français parlé parisien des années 2000 (CFPP2000). cfpp2000.univ-paris3.fr/Corpus.html

Corpus de français parlé au Québec (CFPQ), dir. Gaétane Dostie, CATIFQ, Université de Sherbrooke, 2006. http://re-cherche.flsh.usherbrooke.ca/cfpq/ 
Dagnac A. (2014). « La variation des interrogatives en français », https://hal.archivesouvertes.fr/hal-00988751, document préparatoire pour la GGF (Abeillé \& Godard).

Defrancq B. (2005). L'interrogative enchâssée, Structure et interprétation. Bruxelles : De Boeck Duculot.

Defrancq B. (2000). « Un aspect de la subordination en français parlé : l'interrogation indirecte », Études romanes 47 : 131-141.

Frei H. (1929). La Grammaire des fautes. Genève : Reprints Slatkine.

Gadet F. (éd.) (1997). La variation en syntaxe (= Langue française, 115). Paris : Larousse.

Hadermann P. (1993). Étude morphosyntaxique du mot où. Paris/Louvain-la-Neuve : Duculot.

Karttunen L. (1978). « Syntax and semantics of questions », in Hiz H. (éd.) Questions. Dordrecht :

D. Reidel.

Léard J.-M. (1992). Les Gallicismes, Étude syntaxique et sémantique. Paris/Louvain-la-Neuve : Duculot.

Ledegen G. (2007). «L'interrogative indirecte in situ à la Réunion : elle connaît elle veut quoi », in A. Abecassis et al. (éd.) Le français parlé du XXI siècle : normes et variations géographiques et sociales.

Lefeuvre F. (2015). « Analyse grammaticale et sur corpus de l'expression c'est quoi (?) », in Dostie \& Hadermann (éd.) La dia-variation en français actuel. Études sur corpus, approches croisées et ouvrages de référence. Bern : Peter Lang, coll. Sciences pour la communication, 39-62.

Lefeuvre F. (2006). Quoi de neuf sur quoi ?. Rennes : PUR.

Lefeuvre F. (2009). « Catégorisation de comment subordonnant », Travaux de linguistique, Études sur comment $58: 63-88$.

Lefeuvre F. (2014). Étude grammaticale du français classique à partir de textes. PSN.

Lefeuvre F. \& Rossi-Gensane N. (2015). «Interrogation », in P. Larrivée \& F. Lefeuvre (dir.) Projet Fracov. http://www.univ-paris3.fr/index-des-fiches-227311.kjsp? RH=1373703153287

Le Goffic P. (1993). Grammaire de la phrase française. Paris : Hachette.

Le Goffic P. (1987). "Sur l'ambiguïté des relatives / interrogatives indirectes en ce qui, ce que », in Fuchs (éd.) L'Ambiguïté et la paraphrase : opérations linguistiques, processus cognitifs, traitements automatisés. Centre de publication de l'Université de Caen, 83-88.

Lesort B. (2002). « Entretiens semi-directifs réalisés avec une classe de CM2 », in E. Bautier, S. Branca-Rosoff \& J.-P. Terrail, Décrochage scolaire : genèse et logique des parcours. https:// halshs.archives-ouvertes.fr/halshs-00808806

Martin R. (1985). L'Interrogation comme universel du langage.

Nganang A. P. (2001). Temps de chien. Paris : Serpent à plumes.

Pierrard M. (1989). La Relative sans antécédent. Louvain : Peeters.

Serbat G. (1985). «Le verbe introducteur de la subordonnée interrogative en latin », in Valentin (éd.) L'Interrogation. Presses de l'Université de Paris-Sorbonne, 3-16.

\section{NOTES}

1. Avec le soutien de la Ville de Paris, de la DGLFLF et, récemment, d'Ortolang.

2. Voir Branca-Rosoff, 2015. 
3. Voir les théories de l'accommodation développées tout d'abord par A. Bell, 1984.

4. Ce faible nombre s'explique parce que nous avons préféré transcrire des entretiens longs (souvent de plus d'une heure) qui permettent entre autres de repérer des phénomènes fréquemment attestés chez une personne.

5. Tantôt le locuteur est dans l'ignorance (je ne sais pas quand, où, comment, etc.) ; tantôt, il laisse son interlocuteur dans l'incertitude en ne déterminant pas la valeur de vérité de $\mathrm{p}$ (je sais où, quand, comment...).

6. Cf. également Hadermann, $1993: 126-130$.

7. À l'exception d'un locuteur universitaire qui se reprend après une amorce: « je sais pas qu'est-ce qui te + quel axe euh est pour toi significatif » [SU-01].

8. Héros des Misérables, roman supposé connu de tous les collégiens français.

9. Foulet, 1919, a bien noté qu'en ancien français les formes qu'est-ce que, qui est-ce qui n'étaient pas encore grammaticalisées et qu'elles avaient une valeur d'insistance qui a pu aider à leur diffusion. Le même phénomène se reproduirait pour l'interrogative indirecte.

\section{RÉSUMÉS}

L'article permet de préciser quelques spécificités du corpus CFPP2000 et montre à partir de l'exemple des interrogatives indirectes (percontatives) comment ce corpus peut contribuer à une description de ce que nous appelons «la langue commune». Dans un premier temps, nous rappelons les choix qui ont présidé à la confection du CFPP2000. Puis nous examinons les types d'interrogatives indirectes qui apparaissent dans le CFPP2000, avec des fréquences variées. Les interrogatives indirectes selon la norme grammaticale dominent. Cependant les interrogatives indirectes en « est-ce que " sont bien représentées et elles apparaissent chez des locuteurs de toutes les origines sociales montrant que le corpus CFPP2000 ne se confond pas avec ce standard des grammaires. En revanche, les percontatives in situ sont très rares et plusieurs indices montrent qu'il s'agit encore de formes stigmatisées.

This paper gives some different points about the parisian french spoken corpora (CFPP2000) and shows, from indirect interrogatives clauses ("percontatives"), how this corpora can be exploited for a description of the "common language". First, the choices of the making of the corpora are mentioned. Then the diffents kinds of the indirect interrogatives clauses in the parisian french spoken corpora are evoked. The indirect interrogatives clauses based on grammatical norm are the more frequent. The indirect interrogatives clauses with "est-ce que" occur regularly in the speeches of speakers of all social origins, what shows that the parisian french spoken corpora differs from grammatical norm. On the other hand, the indirect interrogatives clauses in situ are very rare; many clues show that they are stigmatized structures.

\section{INDEX}

Mots-clés : corpus, interrogative indirecte, percontative, langue commune

Keywords : corpus, indirect interrogative, percontative, common language 
AUTEURS

SONIA BRANCA-ROSOFF

SYLED, ED268, Paris 3 Nouvelle Sorbonne

FLORENCE LEFEUVRE

SYLED, ED268, Paris 3 Nouvelle Sorbonne 